\title{
Perspective on the next generation of ultra-low noise fiber supercontinuum sources and their emerging applications in spectroscopy, imaging, and ultrafast photonics
}

\author{
Anupamaa Rampur, ${ }^{1}$ Dirk-Mathys Spangenberg, ${ }^{1}$ Benoît Sierro, ${ }^{1}$ Pascal Hänzi, ${ }^{1}$ Mariusz Klimczak ${ }^{2}$ and \\ Alexander M. Heidt ${ }^{1,}$ a) \\ 1) Institute of Applied Physics, University of Bern, Sidlerstrasse 5, 3012 Bern, Switzerland \\ ${ }^{2)}$ Faculty of Physics, University of Warsaw, Pasteura 5, 02-093 Warsaw, Poland
}

(Dated: 15 June 2021)

\begin{abstract}
A new generation of ultrafast and low-noise supercontinuum (SC) sources is currently emerging, driven by the constantly increasing demands of spectroscopy, advanced microscopy, and ultrafast photonics applications for highly stable broadband coherent light sources. In this perspective, we review recent progress enabled by advances in nonlinear optical fiber design, detail our view on the largely untapped potential for noise control in nonlinear fiber optics, and present the noise fingerprinting technique for measuring and visualizing the noise of SC sources with unprecedented detail. In our outlook we highlight how these SC sources push the boundaries for many spectroscopy and imaging modalities, and focus on their role in the development of ultrafast fiber lasers and frequency combs with ultra-low amplitude and phase noise operating in the $2 \mu \mathrm{m}$ spectral region and beyond in the mid-IR.
\end{abstract}

\section{INTRODUCTION}

Optical spectroscopy and imaging using broadband coherent light sources that cover multiple molecular absorption bands and spectroscopic regimes have enabled fascinating insights into the world of molecular dynamics, revealing how greenhouse gases interact with pollutant particles or the unexpected complexities of water molecule vibrations ${ }^{1,2}$. Supercontinuum (SC) generation based on the nonlinear spectral broadening of high-intensity laser pulses in dispersionengineered specialty optical fibers is a particularly convenient technique for generating broadband coherent light at wavelengths that are otherwise hard to access ${ }^{3}$, and these compact and robust light sources have become indispensable tools in many scientific laboratories. However, due to the everincreasing sensitivity and speed of spectroscopic detection and imaging techniques, the noise properties, shot-to-shot stability and temporal characteristics of fiber-based SC sources are now becoming increasingly relevant.

$\mathrm{SC}$ sources in the visible and near-IR have been commercially available for over a decade, and are the excitation source of choice for a range of advanced microscopy techniques, such as multiphoton- and stimulated emission depletion (STED) microscopy ${ }^{4}$ or optical resolution photoacoustic microscopy ${ }^{5}$. In optical coherence tomography (OCT), SC sources facilitate high-speed, high-resolution 3Dimaging across several medical specialities, including ophthalmology and cardiology ${ }^{6}$, and provide an important diagnostic tool in dermatology for the early-stage detection of skin cancer and other skin diseases ${ }^{7}$. Pumped by high repetitionrate pico- or nanosecond pulsed lasers and equipped with the high beam quality of optical fibers, the brightness of these fiber-based SC sources is unparalleled. Table-top SC sources now outperform synchrotron beamlines in the entire range

\footnotetext{
a)Electronic mail: alexander.heidt@iap.unibe.ch
}

from visible to mid-IR wavelengths, thus removing the necessity of scarce access to expensive facilities, e.g. for IR microspectroscopy applications ${ }^{8}$. However, due to the stochastic nature of the nonlinear processes involved in spectral broadening, these SC sources provide spatially but not temporally coherent light and exhibit very large pulse-to-pulse fluctuations of spectral amplitude and phase ${ }^{9}$. Although the correspondingly large relative intensity noise (RIN) in the order of $50 \%$ can often be reduced by long-term averaging, this SC noise has become the predominating factor limiting acquisition speed, sensitivity, and resolution in many applications ${ }^{10}$.

Pumping with mode-locked femtosecond lasers results in considerably more stable SC that preserve the temporal coherence of the pump pulses, and thus enable the formation of phase-stable broadband frequency combs or ultrashort pulses with only a few optical cycles duration ${ }^{11,12}$. Nevertheless, even in this regime the noise and temporal properties of SC sources are becoming increasingly limiting factors, which are closely linked to the design of the nonlinear fiber used to achieve spectral broadening. Conventional SC sources employ fiber designs with single zero dispersion wavelength (ZDW) closely matched to the central wavelength of the pump pulses, which are injected in the region of anomalous group velocity dispersion (GVD) (Fig. 1 (a)). The resulting coherent nonlinear dynamics dominated by soliton fission generate large spectral bandwidths but rather complex and highly structured spectral and temporal profiles that are mostly unsuitable for ultrafast photonics applications. An additional drawback of this fiber design lies in its facilitation of quantum noise amplification by modulational instability (MI) leading to substantial pulse-to-pulse fluctuations and temporal coherence degradation if it is allowed to dominate, which can only be avoided using very short pump pulse durations and fiber lengths ${ }^{3}$.

Driven by the increased application demand for ultra-low noise broadband coherent light sources, all-normal dispersion (ANDi) SC sources have recently attracted attention as a promising alternative to conventional SC sources. Their 
(a) Conventional SC
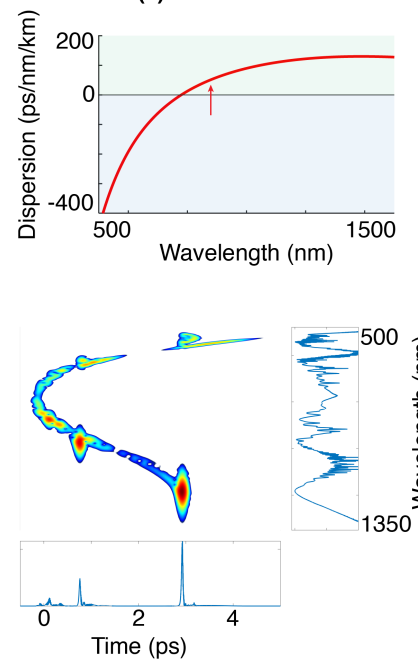

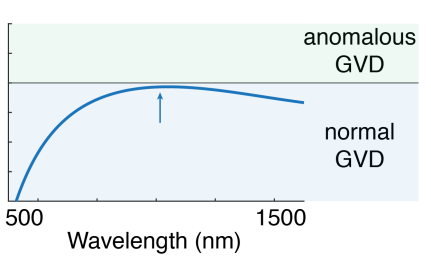

(b) ANDi SC

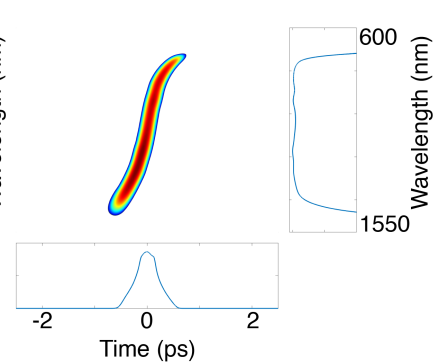

FIG. 1. Properties of typical fiber SC sources pumped by high peak power femtosecond pulses. Fiber dispersion profiles (top) and projected axes spectrogram (bottom) for (a) conventional SC and (b) allnormal dispersion (ANDi) SC. Pump wavelengths are indicated with arrows. Both fiber designs can be adapted for a large range of different pump wavelengths and can cover wavelengths from the UV to the mid-IR. Spectrograms shown here are simulated, but corresponding measurements are available in literature ${ }^{13-15}$.

entirely normal GVD induces coherent nonlinear dynamics dominated by self-phase modulation (SPM) and optical wavebreaking (OWB), which generate flat and smooth spectra of high quality and power density and preserve an ultrashort pulse that is easily recompressed to few- or even single-cycle durations (Fig. 1 (b) ${ }^{16-18}$. While the concept was introduced already over a decade ago, it is now rapidly gaining popularity as it becomes increasingly apparent that the fiber design strongly suppresses the gain for noise-amplifying incoherent nonlinear dynamics ${ }^{19}$.

The renewed interest in low-noise SC sources has generated new insights into the origins of noise during nonlinear spectral broadening and how it can be suppressed using specialty optical fiber design. In this perspective, we detail our view on the largely untapped potential of dispersion engineering for noise control in nonlinear fiber optics and introduce the noise fingerprinting technique, which visualizes for the first time the close correlation between SC noise and the crosssectional geometry of a particular nonlinear fiber. We review the motives behind the recent emergence of highly birefringent ANDi fibers as the key-enabling technology for the next generation of ultra-low noise ultrafast SC sources ${ }^{20}$, and highlight how they are beginning to push the boundaries in several spectroscopy and imaging applications. We further focus on their vital role in the development of ultra-low noise ultrafast fiber lasers and frequency combs operating in the $2 \mu \mathrm{m}$ spectral region and beyond in the mid-IR.

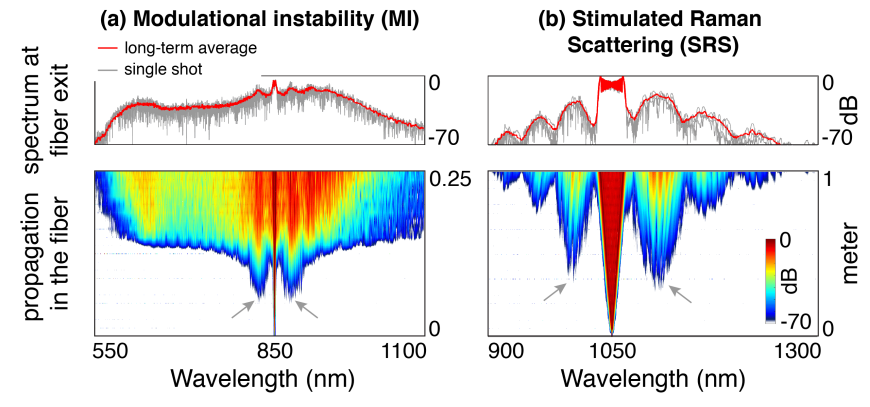

FIG. 2. Origin of SC noise. Simulated spectral evolution of $5 \mathrm{ps}$, $5 \mathrm{~kW}$ pulses in (a) conventional and (b) ANDi fiber from Fig. 1. On top, the mean spectrum is displayed in red, obtained from averaging 20 simulations with random noise seeds. Gray traces show single shot spectra. Arrows indicate primary gain bands for amplification of quantum noise.

\section{ORIGIN OF SUPERCONTINUUM NOISE}

The noise properties of SC sources can be understood in terms of a competition between coherent and incoherent nonlinear processes. Low-noise and phase-coherent SC can be generated in the normal dispersion regime under a wide range of conditions when SPM and OWB dominate ${ }^{19}$, or in the anomalous dispersion regime when soliton fission dominates, i.e. using pump pulses with very short durations and low soliton numbers ${ }^{3}$. However, these coherent dynamics can be disturbed by incoherent nonlinear effects, which can build up new spectral components from quantum noise and whose influence grows with longer pump pulse durations ${ }^{3}$. Examples of this noise amplification are illustrated in Fig. 2 showing SC generated by (a) four-wave mixing (FWM)-induced modulation instabilty (MI) and (b) stimulated Raman scattering (SRS). Both nonlinear effects generate gain bands spectrally separated from the input pulse spectrum, providing enormous exponential gain to any seed signal injected into these bands. When this gain remains unseeded, as in these examples, then random quantum fluctuations serve as the seed and are amplified to become significant and eventually dominate the nonlinear dynamics. The noise-seeded spectral components contained in the MI and SRS peaks exhibit random fluctuations in amplitude and phase from shot to shot and are thus incoherent with the pump. With further propagation, cascaded Stokes (redshifted) and anti-Stokes (blueshifted) gain bands emerge and eventually distribute noise throughout the SC pulse. Similar noise amplifying nonlinear effects exist when coherent and incoherent mode coupling between the two principal polarization axes of a fiber is considered.

Is it possible to control or suppress this nonlinear noise amplification with appropriate fiber design? This question is currently being tackled by several research groups, and here we present our perspective on the effective control of noise in nonlinear fiber optics through suitably designed dispersion profile and fiber transversal geometry. 


\section{NOISE CONTROL BY DISPERSION ENGINEERING}

In order to develop strategies for controlling the stability of SC sources, it is helpful to compare the strength of coherent and incoherent nonlinearities and their dependence on the fiber dispersion profile. The strength of coherent nonlinear dynamics is only weakly dependent on the dispersion regime, as can be concluded from the almost identical expressions for the characteristic length scales of soliton fission and $\mathrm{OWB}^{21,22}$. Therefore, the noise properties of a particular SC source are mainly determined by the gain of incoherent nonlinear effects dominated by MI and SRS. Traditionally, these incoherent dynamics have been treated separately, depending on the fiber dispersion regime; MI is regarded as a dominating mechanism of decoherence in anomalous dispersion ${ }^{23}$. In normal dispersion, MI is usually suppressed, and SRS is considered the predominant effect ${ }^{24}$. Here we provide a different, more comprehensive perspective on noise amplification in nonlinear fiber optics that allows us to unveil the significant potential for control and suppression of noise provided by the well-known concepts of dispersion engineering in specialty optical fibers.

Since the seminal work of Bloembergen and $\operatorname{Shen}^{25}$, it is known that a nonlinear coupling of SRS and FWM occurs in the regime of low dispersion and high nonlinearity, i.e. exactly in the conditions relevant to SC generation. In a single-mode fiber, the interaction between these two nonlinear processes can be described by the mixed parametric Raman (MPR) gain ${ }^{26}$ and given by

$$
g_{M P R}=2 \gamma \Re(\sqrt{K(2 q-K)}),
$$

where $K=-\beta_{2} \Omega_{R}^{2} /\left(2 \gamma P_{0}\right)$ describes the ratio of chromatic dispersion and nonlinearity, with fiber dispersion coefficient $\beta_{2}$ and nonlinear parameter $\gamma$, peak angular frequency shift of the Raman gain $\Omega_{R}$, and pulse peak power $P_{0}$. The factor $q=\left(1-f_{R}\right)+f_{R} \chi_{R}^{(3)}\left(-\Omega_{R}\right)$ contains the Raman susceptibility $\chi_{R}^{(3)}$ and fractional contribution $f_{R}(\sim 0.18$ for silica) of the Raman effect to the total nonlinear response of the fiber material. Fig. 3 shows $g_{M P R}$ for a silica fiber as a function of $K$, normalized to the standard Raman gain coefficient $g_{R} \simeq 0.5 \gamma$.

Eq.(1) describes an explicit dependence of the Raman gain on chromatic dispersion and nonlinearity, and its validity in nonlinear fibers has been experimentally verified ${ }^{27,28}$. However, so far its implications for the control of nonlinear noise amplification by engineering the fiber dispersion profile have not been fully realized. While our recent work has shown the important role of the MPR gain for the noise properties of ANDi $\mathrm{SC}^{19}$, we believe that it can in fact be applied more universally to explain the observed noise properties of SC generated in both dispersion regimes. In Fig. 3 (c), mainly the region $|K|<1$ is relevant to $\mathrm{SC}$ generation, where we observe a strong suppression of the MPR gain in normal dispersion, while MPR-induced noise-amplification is strongly amplified in the anomalous dispersion region. The peak of the MPR gain is located at $K \simeq 1-f_{R} / 2$, where Stokes and anti-Stokes Raman sidebands are effectively amplified by MI, whose role

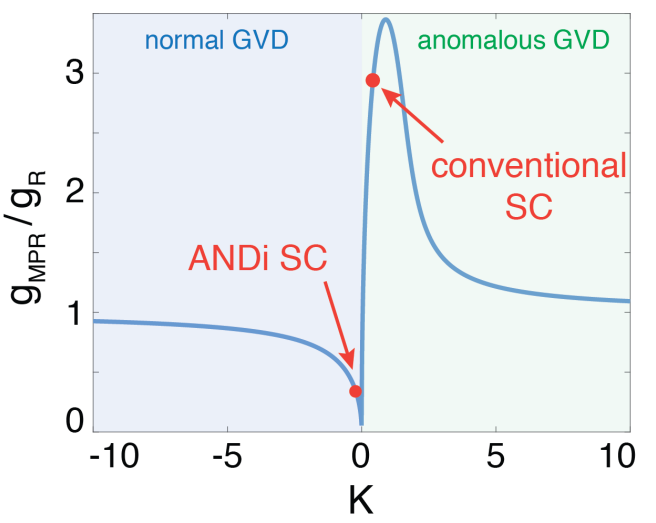

FIG. 3. Mixed parametric Raman (MPR) gain $g_{M P R}$, Eq. (1), normalized by the standard Raman gain $g_{R}$. We use the MPR gain to describe the dispersion dependence of noise-amplifying incoherent nonlinearities in SC generation dynamics. Typical conditions for octave-spanning SC generation for ANDi and conventional SC from Fig. 1 are indicated.

in the coherence collapse of conventional SC has been investigated since the early days of fiber-based SC generation ${ }^{23,29}$. When we consider the typical conditions for octave-spanning SC generation, we find that the MPR gain and associated noise amplification can be decreased by over one order of magnitude by changing from the conventional to the ANDi fiber design. In fact, this order of magnitude difference in noise susceptibility is a recurrent factor found in many theoretical and experimental studies, as we detail below, and can be seen as the main reason behind the attraction of ANDi fibers for low-noise SC source development.

From this fundamental physics perspective, we expect ANDi SC to be significantly more stable than conventional SC. Detailed numerical and experimental studies have confirmed this expectation. The competition between the coherent and incoherent dynamics typically leads to a threshold pulse duration $T_{\text {crit }}$ or threshold soliton number $N_{\text {crit }}$ above which the nature of the SC changes from coherent to incoherent ${ }^{3,30}$. The ANDi fiber design exhibits about 10x higher $T_{c r i t}$ and 50x higher $N_{c r i t}$ than its conventional counterparts for octave-spanning bandwidths ${ }^{19}$. The superior coherence and noise properties of ANDi SCs over conventional SCs were verified, for example, by measurements of relative intensity noise (RIN), spectral coherence, dispersive Fourier transformation, and RF beating with stabilized laser diodes ${ }^{45,51,65}$. ANDi SC also possess a remarkable resistance against technical pump laser fluctuations ${ }^{54,64,66}$, while conventional SCs amplify such fluctuations by up to $20 \mathrm{~dB}$, even in the regime where coherent dynamics dominate ${ }^{62}$. Describing incoherent nonlinear dynamics in terms of the MPR gain is therefore a successful concept for explaining the superior stability of ANDi SC. It also provides a new perspective on the considerable potential for noise control in nonlinear fiber optics. A future challenge will therefore lie in the development of new fiber designs specifically tailored for low-noise operation in a variety of nonlinear frequency conversion applications. 
(a)

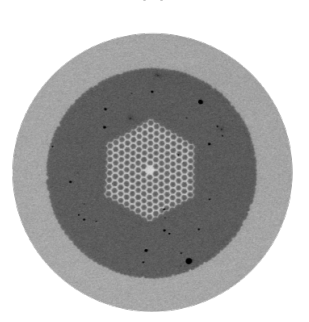

(c)
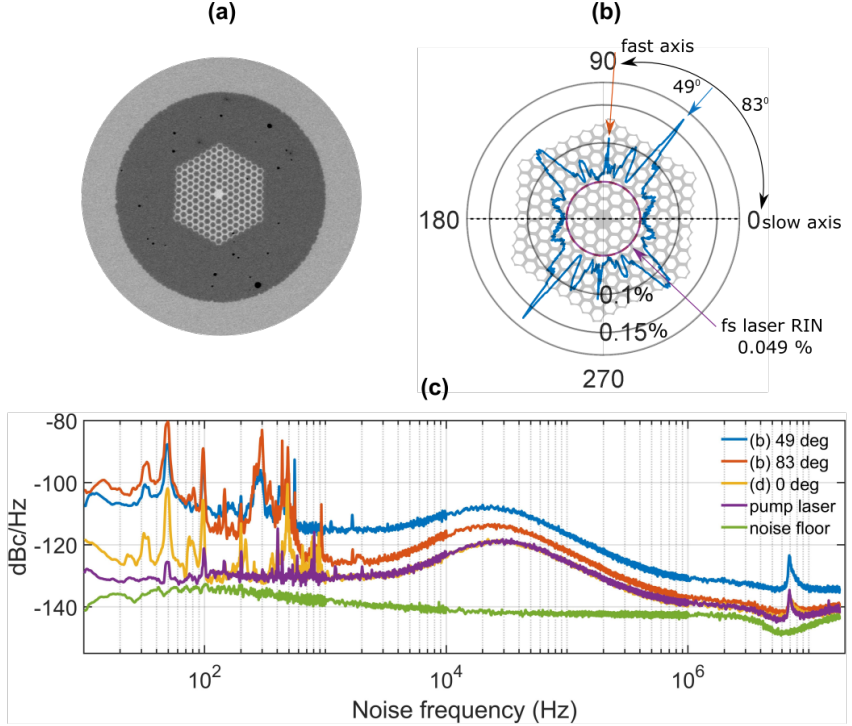

(d)

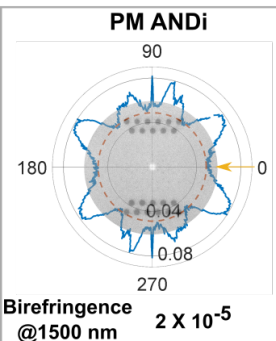

(e)

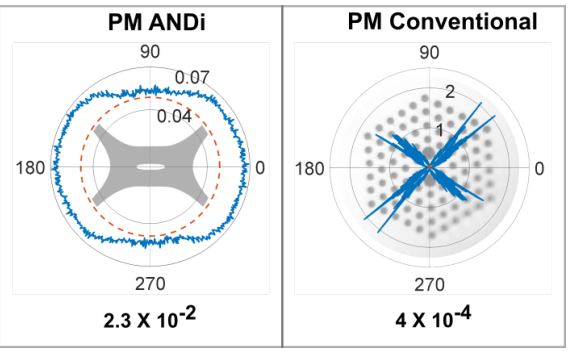

FIG. 4. Noise fingerprints of various ANDi SC (a-e) and conventional SC (f) pumped by an Er:fiber laser ( $80 \mathrm{fs}, 40 \mathrm{MHz}$ ) at $1550 \mathrm{~nm}$, generating comparable bandwidths in the range 1.2-2.2 $\mu \mathrm{m}$. (a) Microscope image of low-birefringence all-solid ANDi photonic crystal fiber $(\mathrm{PCF})^{31}$. (b) Integrated RIN values (in \%) of SC generated in fiber (a) shown in a polar plot in dependence of pump pulse polarization orientation. Corresponding orientation of the fiber geometry shown in background. The fiber's slow axis is aligned to zero degrees. The red-dotted line shows the RIN of the pump laser $(0.05 \%)$. (c) Detailed noise spectra in a range $10 \mathrm{~Hz}-20 \mathrm{MHz}$ for positions $48^{\circ}$ and $83^{\circ}$ (fast axis) of noise fingerprint in (b), and position $0^{\circ}$ (slow axis) of noise fingerprint in (d). Pump laser noise and detection noise floor shown for comparison. (d) Noise fingerprint of SC generated in silica polarization-maintaining (PM) air-hole microstructured $\mathrm{PCF}^{32}$, (e) nanohole suspended-core ANDi fiber ${ }^{33}$, and (f) commercial conventional fiber NKT PM-1550-01.

\section{NOISE CONTROL BY DESIGNING FIBER GEOMETRY AND BIREFRINGENCE}

Every fiber exhibits a certain amount of birefringence that breaks the degeneracy of polarization modes. Birefringence might be induced unintentionally by unavoidable external stresses or bending of the fiber, or it can be engineered, e.g. by including stress rods or asymmetries into the design of the fiber cross-sectional geometry. The coherent and incoherent coupling of the fiber's polarization modes leads to several nonlinear effects that, in addition to the MPR gain, have the potential to amplify quantum noise and result in unpre- dictable fluctuations of the polarization state. In the context of ANDi SC sources especially relevant are polarization modulation instability $(\mathrm{PMI})^{34,35}$, cross-phase modulation instability $(\mathrm{XMI})^{36,37}$, and Raman amplification assisted by cross-phase modulation $^{38,39}$.

In general, the occurrence of noise amplification is a complex function of fiber birefringence and dispersion, as well as relative orientation of input pulse polarization and fiber axes. In Fig. 4 we present preliminary results of high resolution polarization-dependent RIN measurements visualizing this complexity. For these measurements, pulses from an ultrafast Er:fiber laser ( $80 \mathrm{fs}, 40 \mathrm{MHz}, 0.05 \% \mathrm{RIN}$ ) were coupled into ANDi fibers with similar dispersion profiles, but very different geometries and birefringence, generating SC with comparable spectral bandwidths in the range $1.2-2.2$ $\mu \mathrm{m}$. A rotating half-wave plate in front of the fiber and a synchronized analyzer at the fiber exit control the plane of pump pulse and detection polarization with respect to the fiber geometry. Polarization-dependent RIN values are measured with an angular resolution of approximately $0.2^{\circ}$ using a photodiode and electronic spectrum analyzer and visualized in polar plots. These plots were found to be unique for each tested fiber and are therefore referred to as "noise fingerprints".

These measurements reveal a strong correlation between the nonlinear noise amplification and the cross-sectional geometry of a particular fiber, which we attribute to the unique stress profile associated with each fiber structure and its drawing conditions. The ANDi fiber in Fig. 4(a, b) is designed as an all-solid microstructured PCF made from two different soft glasses forming the photonic lattice and inclusions. The structure causes a complex stress pattern due to different thermal expansion coefficients of the two glasses. Since there is no intentional stress axis defined in this design, the resulting birefringence is random and the polarization axes are not well defined, evident by the $83^{\circ}$ angle measured between the axes. The complex stress profile is also reflected in the highly polarization-dependent nonlinear noise amplification pattern, which we found to be highly susceptible to environmental disturbances, such as bending the fiber, different clamping conditions, or day-to-day temperature changes.

Since for each angular RIN measurement a full noise spectrum is available, we can identify the underlying noise amplification process for every feature of the fingerprint by analyzing its characteristic noise frequencies (Fig. 4(c)). For example, at $49^{\circ}$ the noise spectrum of the SC is shifted upward in comparison to the pump laser due to a significant contribution of excess white noise, which is a characteristic signature of quantum noise amplification, and can therefore be attributed to the occurrence of XMI and PMI. Near the fast axis, on the other hand, we mainly observe low-frequency noise, which indicates polarization instability caused by the cancellation of the small linear fiber birefringence by the nonlinear Kerr effect. In general, we observe a complex superposition of these effects.

Fig. 4 also illustrates that birefringence is an effective control parameter to reduce polarization-dependent noise in ANDi SC generation. With increasing birefringence the noise fingerprints become more regular and environmentally sta- 
ble, as shown for the airhole-microstructured silica PCF with Germanium-doped core in Fig. 4 (d). Near the slow axis $\left(0^{\circ}\right)$ of this fiber the noise spectrum is virtually identical to the noise of the pump laser (Fig. 4(c)), while we continue to observe a strong correlation of noise features and fiber geometry for off-axis pumping. Eventually, we observe complete suppression of noise-amplifying nonlinear processes for extremely birefringent ANDi fiber designs, such as the nanohole suspended core fiber in Fig. 4 (e), even when the pump polarization is not aligned to one of the principal fiber axes. In contrast, the noise fingerprint of a comparable polarizationmaintaining conventional SC source (Fig. 4(f)) is significantly more complex, and shows noise amplification up to a factor of 40. In the test conditions the soliton number is $N \simeq 6$, such that a stable SC is generated when the polarization of the pump pulses is exactly aligned to a principal axis of the fiber. However, even slight misalignment of the polarization in the order of just $1^{o}$ causes a significant rise of the SC noise.

These measurements highlight the importance of the crosssectional fiber geometry and the homogeneity of the stress profile, in addition to dispersion engineering, for the realization of high-quality, low-noise SC sources. ANDi SC sources designed with these considerations in mind are currently emerging also for other pump wavelengths, providing further experimental evidence for the excellent quality and stability of these broadband coherent light sources ${ }^{20}$.

\section{APPLICATIONS AND FUTURE DIRECTIONS}

The availability of low-noise ultrafast SC sources has a profound impact on the development of next-generation ultrashort pulse sources and frequency combs operating, for example, in the $2 \mu \mathrm{m}$ spectral region. This waveband is particularly important as stepping stone for the exploration of the molecular fingerprint region in the mid-infrared via nonlinear frequency conversion. As schematically illustrated in Fig. 5(a), such systems are often based on mode-locked Erbium (Er)-fiber systems, which are spectrally broadened to the $2 \mu \mathrm{m}$ region in highly nonlinear fibers and used for the coherent seeding of Thulium (Tm)- or Holmium (Ho)-doped fiber amplifiers ${ }^{40,41}$. This is a convenient approach for extending and power-scaling of Er:fiber technology, which has served as the workhorse for ultrafast photonics and frequency metrology over the past decade and, consequently, has been commercially developed to offer robust, turnkey operation with ultra-low amplitude and phase noise in the $1.55 \mu \mathrm{m}$ window.

However, stable nonlinear spectral broadening dynamics are absolutely critical with this approach, as any noise seed created in the nonlinear fiber will grow exponentially in the subsequent amplifier. In the past, conventional nonlinear fiber designs, often with low-birefringence, were employed in such systems, which made them susceptible to the polarization- and MPR-induced quantum noise amplification discussed above. The resulting RIN values of the amplified output pulse at $2 \mu \mathrm{m}$ were in the range $0.3-0.7 \%$, which corresponds to about an order of magnitude noise amplification compared to the Er:fiber seed $\operatorname{laser}^{40-42}$. This nonlinear noise amplification has re- (a)
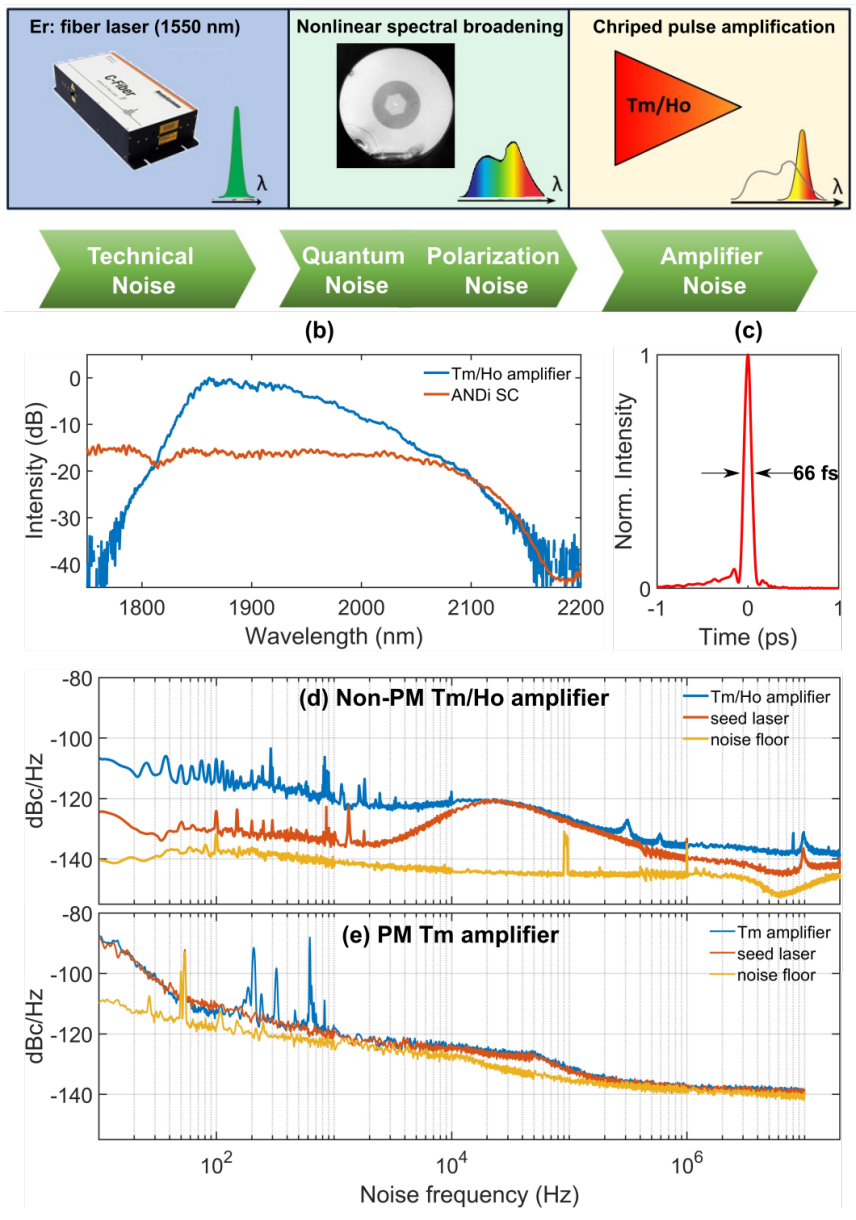

FIG. 5. (a) Schematic overview of extending and power-scaling Er:fiber ultrafast lasers and frequency combs via nonlinear spectral broadening and coherent seeding of Tm- or Ho:fiber amplifiers operating in the $2 \mu \mathrm{m}$ spectral region. Sources of excess noise of each stage are also indicated. (b) Measured spectrum of a Tm/Ho-codoped chirped pulse fiber amplifier seeded by ANDi SC. (c) Measured pulse at the output of the all-fiber system. Noise spectra of amplified pulses in a system built with low-birefringence fibers (d) and after upgrade of the entire system to PM fibers (e). (b)-(d) Adapted from Heidt et al., Sci. Rep. 10, $16734(2020)^{43}$; licensed under a Creative Commons Attribution (CC BY) license. (e) Adapted with permission from Rampur et al., Opt. Express 27, 35041 (2019) ${ }^{44}$. Copyright 2019 The Optical Society.

cently been identified as the major performance limiting factor in the further development of high-power frequency comb sources at $2 \mu \mathrm{m}^{42}$.

In recent proof-of-principle experiments we demonstrated that making use of ANDi fibers in the nonlinear broadening stage overcomes these limitations and significantly reduces nonlinear noise amplification. A $2 \mu \mathrm{m} \mathrm{Tm} / \mathrm{Ho}$-codoped chirped pulse fiber amplifier system coherently seeded by an ANDi SC was realized ${ }^{43}$. Fig. 5 (b) shows its spectrum with a $-20 \mathrm{~dB}$ bandwidth of $320 \mathrm{~nm}$ and the comparison to the ANDi SC seed. High quality 66 fs pulses with $70 \mathrm{~kW}$ peak power could be obtained at the output of the all-fiber sys- 
tem without employing any additional pulse shortening techniques (Fig. 5 (c)). Due to the suppression of MPR gain in ANDi fibers, the amplified pulses exhibit a RIN of only $0.07 \%$. However, the noise frequency spectrum shown in Fig. 5(d) still shows significantly elevated noise in comparison to the Er:fiber seed laser, crucially also at high frequencies, which is a signature of PMI and XMI occurring in the lowbirefringence fibers used in this first implementation. With the upgrade of the complete system to highly birefringent polarization-maintaining fibers any type of nonlinear noise amplification could be suppressed, as evident from Fig. 5 (c), and the amplifier RIN lowered to $0.03 \%$, virtually identical to the Er:fiber seed laser ${ }^{44}$. These experiments also proved that in addition to the suppression of incoherent nonlinearities, the coherent seeding of the entire amplifier gain spectrum with the broadband ANDi SC also effectively suppresses amplified spontaneous emission noise in the amplifier. In combination, the results represent an order of magnitude improvement of amplifier noise over conventionally seeded implementations.

Recent experiments convincingly demonstrated the advantages of ANDi SC over conventional SC in the construction of stabilized frequency combs ${ }^{45}$. In combination with the progress of fiber amplifiers described above, these studies have laid the foundations for exciting opportunities arising from using ANDi SC seed sources for the next generation of ultra-low noise frequency combs and ultrafast fiber amplifiers operating in the $2 \mu \mathrm{m}$ spectral region, which are currently of tremendous interest for extending the coverage of frequency combs to the molecular fingerprint region $(3-20 \mu \mathrm{m})$. Two pathways seem particularly attractive for reaching the mid-IR:

(i) parametric downconversion in mid-IR transparent nonlinear crystals, which are mostly opaque at $1 \mu \mathrm{m}$ wavelength, where high power driving lasers would be readily available. Hence, an urgent demand exists for the development of powerful, ultra-low-noise ultrafast fiber laser systems at wavelengths $>1.5 \mu \mathrm{m}^{46}$. The spectral broadening in PM-ANDi fibers discussed in this perspective letter should in future allow the straightforward power scaling of Er:fiber frequency combs in Tm- and Ho:fiber or even Er:ZBLAN amplifiers without significant noise penalties.

(ii) SC generation in mid-IR transparent ANDi fibers, e.g. made from chalcogenide glasses, which have seen a fastpaced development in recent years and have achieved spectral coverage up to $12 \mu \mathrm{m}^{47}$. A remaining challenge is the realization of highly birefringent fiber designs that can stabilize the polarization state of the SC over such a broad spectral region and avoid polarization-induced quantum-noise amplification. Circular birefringence induced by intentional spinning of the preform during fiber drawing was recently shown to stabilize the polarization state of near-IR SC sources and is a particularly elegant concept to extend to the mid-IR ${ }^{48}$. The helical twisting adds another dimension and degree of freedom to the fiber design process and could be simpler to realize than comparable methods of controlling linear birefringence when working with technologically less mature soft glass materials. Stable and temporally recompressible ANDi SC generation in the mid-IR is a promising approach to realize all-fiber singlecycle pulse sources, widely tunable femtosecond lasers, and ultra-low noise phase-stable frequency combs in this emerging waveband ${ }^{47,49-51}$.

While our discussion has so far focused on intensity noise, nonlinear spectral broadening in the normal dispersion regime also has substantial advantages when phase noise or relative timing jitter (RTJ) between the spectral components is considered. ANDi SC generate new spectral components that are temporally synchronized to the pump pulse with RTJ in the order of only 100 attoseconds, while conventional SC exhibit up to 2 orders of magnitude larger fluctuations ${ }^{52-54}$. This is due to the coupling of amplitude and phase noise during soliton propagation in anomalous GVD, which is significantly reduced in SPM-dominated spectral broadening in normal GVD. This tight passive temporal synchronization on time scales much shorter than an optical cycle enables exciting prospects that have barely been exploited, e.g. for twocolour pump-probe spectroscopy, coherent combining, or optical parametric chirped pulse amplification.

Low-noise ultrafast ANDi SC sources have also started to push the boundaries in several spectroscopy and imaging modalities. Their recent implementation in hyperspectral SRS microscopy for label-free, chemical-specific biomedical and mineralogical imaging is particularly impressive, as source noise is critically important in this technique and has excluded the use of many other nonlinear spectral broadening schemes $^{55}$. In high-resolution spectral-domain OCT ANDi SC sources have led to a paradigm shift, where image quality is no longer limited by SC source noise but by detection shot-noise $^{56}$. ANDi SC are also starting to replace white-light generation in bulk media like YAG, sapphire or $\mathrm{CaF}_{2}$ in ultrafast spectroscopy, removing the need for expensive amplified laser systems ${ }^{57}$. In combination with digital pulse shaping and ptychographic algorithms, they facilitate significant signalto-noise and thus speed improvements in single-beam coherent anti-Stokes Raman scattering (CARS) micro-spectroscopy and multiphoton imaging ${ }^{58,59}$. The combination of multiphoton microscopy and hyperspectral CARS imaging based on digitally programmable ANDi SC pulses has resulted in an extremely versatile platform for biomedical imaging, offering the potential to translate label-free molecular histopathology for disease diagnosis into routine clinical use ${ }^{60}$. Even without the relatively expensive digital pulse shaping device, broadband multimodal CARS and multi-photon imaging systems based on ANDi SC sources have recently been demonstrated $^{61}$. From our perspective this is really just the beginning of a movement that will see these versatile sources of broadband, low-noise, and ultrashort pulses being used in an increasing numbers of applications which so far were not able to use conventional fiber-based SC sources due to their noise or complex temporal pulse shapes.

In nonlinear fiber optics, dispersion engineering has been a highly successful concept to control nonlinear effects and tailor the properties of a particular light source to application demands. However, this approach has so far mainly focused on coherent dynamics. Its full potential to control or suppress noise amplification by influencing the gain of incoherent dynamics is yet to be explored. Based on the concepts presented in this perspective, we expect the development of new fiber 
designs specifically tailored to suppress noise in a multitude of nonlinear wavelength conversion processes. Effective noise engineering will also require the consideration of the fiber cross-sectional geometry and stress profile. Highly birefringent fiber designs will probably play a more important role in the future as they seem to inhibit most noise-amplifying nonlinear effects arising from coherent or incoherent coupling of the polarization modes. It is also worth reconsidering longitudinally varying dispersion designs, e.g. by slow tapering of the fiber and adjusting the taper rate for minimizing MPR gain and related build-up of noise. This adds another dimension to the fiber design and enables a variety of innovative hybrid approaches, which exploit both enhanced spectral broadening in anomalous GVD as well as enhanced stability in the normal GVD. While theoretical studies have investigated such designs already over a decade ago $^{30}$, their experimental realization would be a logical next step given today's substantially increased interest in low-noise SC sources.

\section{DATA AVAILABILITY}

The data that support the findings of this study are openly available in the Bern Open Research Information System (BORIS) at http://dx.doi.org/10.48350/156485.

\section{ACKNOWLEDGMENTS}

We thank A. Hartung, K. Tarnowski, P. Mergo, and T. Martynkien for supplying fiber samples for the noise fingerprinting. Funding from the Swiss National Science Foundation (PCEFP2_181222) and Fundacja na rzecz Nauki Polskiej (FNP) (First TEAM/2016-1/1, POIR.04.04.00-00-1D64/1600) is gratefully acknowledged.

${ }^{1}$ C. J. Ostaszewski, N. M. Stuart, D. M. Lesko, D. Kim, M. J. Lueckheide, and J. G. Navea, "Effects of coadsorbed water on the heterogeneous photochemistry of nitrates adsorbed on $\mathrm{TiO}_{2}$," J. Phys. Chem. A 122, 6360-6371 (2018).

${ }^{2}$ K. Ramasesha, L. De Marco, A. Mandal, and A. Tokmakoff, "Water vibrations have strongly mixed intra-and intermolecular character," Nature Chemistry 5, 935-940 (2013).

${ }^{3}$ J. M. Dudley, G. Genty, and S. Coen, "Supercontinuum generation in photonic crystal fiber," Rev. Mod. Phys. 78, 1135-1184 (2006).

${ }^{4}$ D. Wildanger, E. Rittweger, L. Kastrup, and S. W. Hell, "STED microscopy with a supercontinuum laser source," Opt. Express 16, 9614-9621 (2008).

${ }^{5}$ M. K. Dasa, G. Nteroli, P. Bowen, G. Messa, Y. Feng, C. R. Petersen, S. Koutsikou, M. Bondu, P. M. Moselund, A. Podoleanu, et al., "All-fibre supercontinuum laser for in vivo multispectral photoacoustic microscopy of lipids in the extended near-infrared region," Photoacoustics 18, 100163 (2020).

${ }^{6}$ S. Moon and D. Y. Kim, "Ultra-high-speed optical coherence tomography with a stretched pulse supercontinuum source," Opt. Express 14, 1157511584 (2006).

${ }^{7}$ N. M. Israelsen, M. Maria, M. Mogensen, S. Bojesen, M. Jensen, M. Haedersdal, A. Podoleanu, and O. Bang, "The value of ultrahigh resolution OCT in dermatology - delineating the dermo-epidermal junction, capillaries in the dermal papillae and vellus hairs," Biomed. Opt. Express 9, 2240-2265 (2018).

${ }^{8}$ C. R. Petersen, P. M. Moselund, L. Huot, L. Hooper, and O. Bang, "Towards a table-top synchrotron based on supercontinuum generation," Infrared Phys. Technol. 91, 182-186 (2018).
${ }^{9}$ S. Dupont, Z. Qu, S.-S. Kiwanuka, L. E. Hooper, J. C. Knight, S. R. Keiding, and C. F. Kaminski, "Ultra-high repetition rate absorption spectroscopy with low noise supercontinuum radiation generated in an allnormal dispersion fibre," Laser Phys. Lett. 11, 075601 (2014).

${ }^{10}$ M. Jensen, I. B. Gonzalo, R. D. Engelsholm, M. Maria, N. M. Israelsen, A. Podoleanu, and O. Bang, "Noise of supercontinuum sources in spectral domain optical coherence tomography," J. Opt. Soc. Am. B 36, A154-A160 (2019).

${ }^{11}$ T. Udem, R. Holzwarth, and T. W. Hänsch, "Optical frequency metrology," Nature 416, 233-237 (2002).

${ }^{12}$ J. Rothhardt, S. Hädrich, J. Delagnes, E. Cormier, and J. Limpert, "High average power near-infrared few-cycle lasers," Laser Photonics Rev. 11 (2017).

${ }^{13}$ A. M. Heidt, D.-M. Spangenberg, M. Brügmann, E. G. Rohwer, and T. Feurer, "Improved retrieval of complex supercontinuum pulses from XFROG traces using a ptychographic algorithm," Opt. Lett. 41, 4903-4906 (2016).

${ }^{14}$ A. Okamura, Y. Sakakibara, E. Omoda, H. Kataura, and N. Nishizawa, "Experimental analysis of coherent supercontinuum generation and ultrashort pulse generation using cross-correlation frequency resolved optical gating (X-FROG)," J. Opt. Soc. Am. B 32, 400-406 (2015).

${ }^{15}$ A. Rampur, D.-M. Spangenberg, G. Stepniewski, D. Dobrakowski, K. Tarnowski, K. Stefanska, A. Pazdzior, P. Mergo, T. Martynkien, T. Feurer, et al., "Temporal fine structure of all-normal dispersion fiber supercontinuum pulses caused by non-ideal pump pulse shapes," Opt. Express 28, 16579-16593 (2020).

${ }^{16}$ T. Hori, J. Takayanagi, N. Nishizawa, and T. Goto, "Flatly broadened, wideband and low noise supercontinuum generation in highly nonlinear hybrid fiber," Opt. Express 12, 317-324 (2004).

${ }^{17}$ A. M. Heidt, "Pulse preserving flat-top supercontinuum generation in allnormal dispersion photonic crystal fibers," J. Opt. Soc. Am. B 27, 550-559 (2010).

${ }^{18}$ A. M. Heidt, A. Hartung, and H. Bartelt, "The supercontinuum laser source: The ultimate white light," (Springer, 2016) Chap. Generation of Ultrashort and Coherent Supercontinuum Light Pulses in All-Normal Dispersion Fibers, pp. 247-280.

${ }^{19}$ A. M. Heidt, J. S. Feehan, J. H. V. Price, and T. Feurer, "Limits of coherent supercontinuum generation in normal dispersion fibers," J. Opt. Soc. Am. B 34, 764-775 (2017).

${ }^{20}$ E. Genier, S. Grelet, R. D. Engelsholm, P. Bowen, P. M. Moselund, O. Bang, J. M. Dudley, and T. Sylvestre, "An ultra-flat, low-noise and linearly polarized fiber supercontinuum source covering $670 \mathrm{~nm}-1390 \mathrm{~nm}$," Opt. Lett. 46, 1820-1823 (2021).

${ }^{21}$ C.-M. Chen and P. L. Kelley, "Nonlinear pulse compression in optical fibers: scaling laws and numerical analysis," J. Opt. Soc. Am. B 19, 19611967 (2002).

${ }^{22}$ C. Finot, B. Kibler, L. Provost, and S. Wabnitz, "Beneficial impact of wavebreaking for coherent continuum formation in normally dispersive nonlinear fibers," J. Opt. Soc. Am. B 25, 1938-1948 (2008).

${ }^{23}$ M. Nakazawa, K. Tamura, H. Kubota, and E. Yoshida, "Coherence degradation in the process of supercontinuum generation in an optical fiber," Opt. Fiber Technol. 4, 215-223 (1998).

${ }^{24} \mathrm{U}$. Møller and O. Bang, "Intensity noise in normal-pumped picosecond supercontinuum generation, where higher-order Raman lines cross into anomalous dispersion regime," Electron. Lett. 49, 63-65 (2013).

${ }^{25} \mathrm{~N}$. Bloembergen and Y. R. Shen, "Coupling between vibrations and light waves in Raman laser media," Phys. Rev. Lett. 12, 504-507 (1964).

${ }^{26}$ S. Coen, A. H. L. Chau, R. Leonhardt, J. D. Harvey, J. C. Knight, W. J. Wadsworth, and P. S. J. Russell, "Supercontinuum generation by stimulated Raman scattering and parametric four-wave mixing in photonic crystal fibers," J. Opt. Soc. Am. B 19, 753-764 (2002).

${ }^{27}$ S. Coen, D. A. Wardle, and J. D. Harvey, "Observation of non-phasematched parametric amplification in resonant nonlinear optics," Phys. Rev. Lett. 89, 273901 (2002).

${ }^{28}$ F. Vanholsbeeck, P. Emplit, and S. Coen, "Complete experimental characterization of the influence of parametric four-wave mixing on stimulated Raman gain," Opt. Lett. 28, 1960 (2003).

${ }^{29}$ K. L. Corwin, N. R. Newbury, J. M. Dudley, S. Coen, S. A. Diddams, K. Weber, and R. S. Windeler, "Fundamental noise limitations to supercontinuum generation in microstructure fiber," Phys. Rev. Lett. 90, 113904 
(2003).

${ }^{30}$ G. Genty, S. Coen, and J. M. Dudley, "Fiber supercontinuum sources (invited)," J. Opt. Soc. Am. B 24, 1771-1785 (2007).

${ }^{31}$ M. Klimczak, B. Siwicki, A. Heidt, and R. Buczyński, "Coherent supercontinuum generation in soft glass photonic crystal fibers," Photonics Res. 5, 710-727 (2017).

${ }^{32}$ K. Tarnowski, T. Martynkien, P. Mergo, K. Poturaj, A. Anuszkiewicz, P. Béjot, F. Billard, O. Faucher, B. Kibler, and W. Urbanczyk, "Polarized all-normal dispersion supercontinuum reaching $2.5 \mu \mathrm{m}$ generated in a birefringent microstructured silica fiber," Opt. Express 25, 27452-27463 (2017).

${ }^{33}$ A. Hartung, J. Bierlich, A. Lorenz, J. Kobelke, and M. Jäger, "Design and fabrication of all-normal dispersion nanohole suspended-core fibers," J. Opt. Soc. Am. B 36, 3404-3410 (2019).

${ }^{34}$ Y. Liu, Y. Zhao, J. Lyngs $\varnothing$, S. You, W. L. Wilson, H. Tu, and S. A. Boppart, "Suppressing short-term polarization noise and related spectral decoherence in all-normal dispersion fiber supercontinuum generation," J. Lightwave Technol. 33, 1814-1820 (2015).

${ }^{35}$ I. B. Gonzalo, R. D. Engelsholm, M. P. Sørensen, and O. Bang, "Polarization noise places severe constraints on coherence of all-normal dispersion femtosecond supercontinuum generation," Sci. Rep. 8, 1-13 (2018).

${ }^{36}$ G. P. Agrawal, P. L. Baldeck, and R. R. Alfano, "Modulation instability induced by cross-phase modulation in optical fibers," Phys. Rev. A 39, 3406-3413 (1989).

${ }^{37}$ E. Genier, A. N. Ghosh, S. Bobba, P. Bowen, P. M. Moselund, O. Bang, J. M. Dudley, and T. Sylvestre, "Cross-phase modulation instability in PM ANDi fiber-based supercontinuum generation," Opt. Lett. 45, 3545-3548 (2020).

${ }^{38}$ J. S. Feehan and J. H. V. Price, "Decoherence due to XPM-assisted Raman amplification for polarization or wavelength offset pulses in all-normal dispersion supercontinuum generation," J. Opt. Soc. Am. B 37, 635-644 (2020).

${ }^{39}$ J. S. Feehan, E. Brunetti, S. Yoffe, W. Li, S. M. Wiggins, D. A. Jaroszynski, and J. H. V. Price, "Noise-related polarization dynamics for femto and picosecond pulses in normal dispersion fibers," Opt. Express 28, 2144721463 (2020).

${ }^{40}$ F. Adler and S. A. Diddams, "High-power, hybrid Er:fiber/Tm:fiber frequency comb source in the $2 \mu \mathrm{m}$ wavelength region," Opt. Lett. 37, 14001402 (2012).

${ }^{41}$ H. Hoogland, A. Thai, D. Sánchez, S. Cousin, M. Hemmer, M. Engelbrecht, J. Biegert, and R. Holzwarth, "All-PM coherent $2.05 \mu \mathrm{m}$ Thulium/Holmium fiber frequency comb source at $100 \mathrm{MHz}$ with up to $0.5 \mathrm{~W}$ average power and pulse duration down to $135 \mathrm{fs}$," Opt. Express 21, 31390-31394 (2013).

${ }^{42}$ C. Gaida, T. Heuermann, M. Gebhardt, E. Shestaev, T. P. Butler, D. Gerz, N. Lilienfein, P. Sulzer, M. Fischer, R. Holzwarth, A. Leitenstorfer, I. Pupeza, and J. Limpert, "High-power frequency comb at $2 \mu \mathrm{m}$ wavelength emitted by a Tm-doped fiber laser system," Opt. Lett. 43, 5178-5181 (2018).

${ }^{43}$ A. M. Heidt, J. M. Hodasi, A. Rampur, D.-M. Spangenberg, M. Ryser, M. Klimczak, and T. Feurer, "Low noise all-fiber amplification of a coherent supercontinuum at $2 \mu \mathrm{m}$ and its limits imposed by polarization noise," Sci. Rep. 10, 16734 (2020).

${ }^{44}$ A. Rampur, Y. Stepanenko, G. Stępniewski, T. Kardaś, D. Dobrakowski, D.-M. Spangenberg, T. Feurer, A. Heidt, and M. Klimczak, "Ultra lownoise coherent supercontinuum amplification and compression below 100 fs in an all-fiber polarization-maintaining thulium fiber amplifier," Opt. Express 27, 35041 (2019).

${ }^{45}$ N. Nishizawa, T. Niinomi, Y. Nomura, L. Jin, and Y. Ozeki, "Octave spanning coherent supercontinuum comb generation based on Er-doped fiber lasers and their characterization," IEEE J. Sel. Topics Quantum Electron. 24, 1-9 (2018).

${ }^{46}$ D. M. Lesko, H. Timmers, S. Xing, A. Kowligy, A. J. Lind, and S. A. Diddams, "A six-octave optical frequency comb from a scalable few-cycle erbium fibre laser," Nat. Photonics , 1-6 (2021).

${ }^{47}$ N. Zhang, X. Peng, Y. Wang, S. Dai, Y. Yuan, J. Su, G. Li, P. Zhang, P. Yang, and X. Wang, "Ultrabroadband and coherent mid-infrared supercontinuum generation in Te-based chalcogenide tapered fiber with allnormal dispersion,” Opt. Express 27, 10311-10319 (2019).
${ }^{48}$ R. P. Sopalla, G. K. Wong, N. Y. Joly, M. H. Frosz, X. Jiang, G. Ahmed, and P. S. J. Russell, "Generation of broadband circularly polarized supercontinuum light in twisted photonic crystal fibers," Opt. Lett. 44, 3964-3967 (2019).

${ }^{49}$ J. H. Price, X. Feng, A. M. Heidt, G. Brambilla, P. Horak, F. Poletti, G. Ponzo, P. Petropoulos, M. Petrovich, J. Shi, M. Ibsen, W. H. Loh, H. N. Rutt, and D. J. Richardson, "Supercontinuum generation in nonsilica fibers," Opt. Fiber Technol. 18, 327 - 344 (2012).

${ }^{50}$ K. F. Lee, N. Granzow, M. A. Schmidt, W. Chang, L. Wang, Q. Coulombier, J. Troles, N. Leindecker, K. L. Vodopyanov, P. G. Schunemann, et al., "Midinfrared frequency combs from coherent supercontinuum in chalcogenide and optical parametric oscillation," Opt. Lett. 39, 2056-2059 (2014).

${ }^{51}$ M. Klimczak, B. Siwicki, B. Zhou, M. Bache, D. Pysz, O. Bang, and R. Buczyński, "Coherent supercontinuum bandwidth limitations under femtosecond pumping at $2 \mu \mathrm{m}$ in all-solid soft glass photonic crystal fibers," Opt. Express 24, 29406-29416 (2016).

${ }^{52}$ Y. Hua, G. Zhou, W. Liu, M. Xin, F. X. Kärtner, and G. Chang, "Femtosecond two-color source synchronized at 100-as-precision based on SPMenabled spectral selection," Opt. Lett. 45, 3410-3413 (2020).

${ }^{53}$ J. Rothhardt, A. M. Heidt, S. Hädrich, S. Demmler, J. Limpert, and A. Tünnermann, "High stability soliton frequency-shifting mechanisms for laser synchronization applications," J. Opt. Soc. Am. B 29, 1257-1262 (2012).

${ }^{54}$ B. Sierro and A. Heidt, "Noise amplification in all-normal dispersion fiber supercontinuum generation and its impact in ultrafast photonics applications," OSA Continuum 3, 2347-2361 (2020).

${ }^{55}$ P. Abdolghader, A. F. Pegoraro, N. Y. Joly, A. Ridsdale, R. Lausten, F. Légaré, and A. Stolow, "All normal dispersion nonlinear fibre supercontinuum source characterization and application in hyperspectral stimulated raman scattering microscopy," Opt. Express 28, 35997-36008 (2020).

${ }^{56}$ S. Rao D. S., M. Jensen, L. Grüner-Nielsen, J. T. Olsen, P. Heiduschka, B. Kemper, J. Schnekenburger, M. Glud, M. Mogensen, N. M. Israelsen, and O. Bang, "Shot-noise limited, supercontinuum based optical coherence tomography,' Light Sci. Appl. , (in press) (2021).

${ }^{57}$ N. M. Kearns, A. C. Jones, M. B. Kunz, R. T. Allen, J. T. Flach, and M. T. Zanni, "Two-dimensional white-light spectroscopy using supercontinuum from an all-normal dispersion photonic crystal fiber pumped by a $70 \mathrm{MHz}$ Yb fiber oscillator," J. Phys. Chem. A 123, 3046-3055 (2019).

${ }^{58}$ R. Viljoen, P. Neethling, D. Spangenberg, A. Heidt, H.-M. Frey, T. Feurer, and E. Rohwer, "Implementation of temporal ptychography algorithm, $\mathrm{i}^{2} \mathrm{PIE}$, for improved single-beam coherent anti-Stokes Raman scattering measurements," J. Opt. Soc. Am. B 37, A259-A265 (2020).

${ }^{59}$ G. Dwapanyin, D. Spangenberg, A. Heidt, T. Feurer, G. Bosman, P. Neethling, and E. Rohwer, "Generalized spectral phase-only time-domain ptychographic phase reconstruction applied in nonlinear microscopy," J. Opt. Soc. Am. B 37, A285-A292 (2020).

${ }^{60}$ H. Tu, Y. Liu, D. Turchinovich, M. Marjanovic, J. K. Lyngsø, J. Lægsgaard, E. J. Chaney, Y. Zhao, S. You, W. L. Wilson, et al., "Stain-free histopathology by programmable supercontinuum pulses," Nat. Photonics 10, 534 (2016).

${ }^{61}$ K. P. Herdzik, K. N. Bourdakos, P. B. Johnson, A. P. Lister, A. P. Pitera, C.-y. Guo, P. Horak, D. J. Richardson, J. H. Price, and S. Mahajan, "Multimodal spectral focusing CARS and SFG microscopy with a tailored coherent continuum from a microstructured fiber," Appl. Phys. B 126, 84 (2020).

${ }^{62}$ N. R. Newbury, B. R. Washburn, K. L. Corwin, and R. S. Windeler, "Noise amplification during supercontinuum generation in microstructure fiber," Opt. Lett. 28, 944-946 (2003).

${ }^{63}$ E. Genier, P. Bowen, T. Sylvestre, J. M. Dudley, P. Moselund, and O. Bang, "Amplitude noise and coherence degradation of femtosecond supercontinuum generation in all-normal-dispersion fibers," J. Opt. Soc. Am. B 36, A161-A167 (2019).

${ }^{64}$ S. Rao D. S., R. D. Engelsholm, I. B. Gonzalo, B. Zhou, P. Bowen, P. M. Moselund, O. Bang, and M. Bache, "Ultra-low-noise supercontinuum generation with a flat near-zero normal dispersion fiber," Opt. Lett. 44, 22162219 (2019).

${ }^{65}$ N. Nishizawa and J. Takayanagi, "Octave spanning high-quality supercontinuum generation in all-fiber system,” J. Opt. Soc. Am. B 24, 1786-1792 (2007).

${ }^{66}$ Z. Eslami, P. Ryczkowski, L. Salmela, and G. Genty, "Low-noise octavespanning mid-infrared supercontinuum generation in a multimode chalcogenide fiber," Opt. Lett. 45, 3103-3106 (2020). 
${ }^{67}$ M. Klimczak, G. Soboń, R. Kasztelanic, K. M. Abramski, and R. Buczyński, "Direct comparison of shot-to-shot noise performance of all normal dispersion and anomalous dispersion supercontinuum pumped with sub-picosecond pulse fiber-based laser," Sci. Rep. 6, 19284 (2016)

${ }^{68}$ J. Olsen, J. Holmes, and G. B. E. Jemec, "Advances in optical coherence tomography in dermatology—a review," J. Biomed. Opt. 23, 040901 (2018). 